\title{
COMPARISON OF VISCOUS AND INVISCID NUMERICAL SIMULATIONS OF THE START-UP VORTEX ISSUING FROM A SEMI-INFINITE FLAT PLATE
}

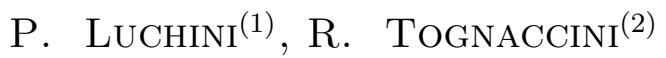 \\ (1) Dipartimento di Ingegneria Aerospaziale, \\ Politecnico di Milano, Italia, luchini@aero.polimi.it \\ ${ }^{(2)}$ Dipartimento di Progettazione Aeronautica, \\ Universita' di Napoli "Federico II", Italia, rtogna@unina.it
}

\begin{abstract}
Subject of the present work is the start-up vortex issuing from a sharp trailing edge accelerated from rest in still air. A numerical simulation of the flow has been performed in case of a semi-infinite flat plate by solving the Navier-Stokes equations in the $\psi-\omega$ formulation. The new numerical algorithm is based on a fast multigrid implicit integration of the difference equations in an unstructured mesh that is dynamically built to minimize the computational costs. The results, compared with inviscid lumped-vortex simulations, show the appearance of instabilities that quickly destroy the regular structure of the spiral vortex. This analysis gives a contribution to the understanding of a physical problem still discussed in the literature and raises the question whether the application of numerical regularization procedures can tend to stabilize a phenomenon even when its physical nature is unstable.
\end{abstract}

\section{Introduction}

The idea that the initial evolution of the start-up vortex shed by a sharp edge might be described by a class of self-similar solutions of the Euler equations dates back to Prandtl, but the determination of such a motion proved non trivial, and its stability was questioned by several authors. In 1931 Kaden introduced similarity variables for the roll-up of a semi-infinite plane vortex sheet [1]; during the fifties, several authors presented approximate asymptotic solutions in which the spiral vortex generated behind a sharp edge was replaced by a single point vortex, an example of which is given by Rott [2]. Although Moore [3] had proved the stability of certain stretching spiral vortices, Pullin [4] noted that Moore's demonstration did not fully apply to the start-up problem. Nevertheless, in the same work, he proposed numerical solutions of regular and well defined start-up vortex spirals obtained by solving the Birkhoff-Rott equation written in similarity variables and without an explicit presence of the time variable. Moreover, the stability of this class of flows was not clarified by experiments that, performed by different authors, proposed different conclusions, Pierce [5], Pullin and Perry [6].

Luchini, among others, studied this phenomenon by computing the inviscid vortex shedding from a semi-infinite wedge [7] and from an airfoil [8]. The incompressible time-dependent Euler equations were solved by a lumped-vortex formulation. The results showed the presence of chaotic oscillations of the vortex structure ever since infinitesimal time, thus questioning the generally adopted assumption of a regular vortex sheet as an initial condition. Preliminary viscous computations 
[9] were in qualitative agreement with the inviscid results. Recently [10], solutions of the viscous start-up problem for a plate of finite length by a "blob" method have been proposed. On the basis of their results the authors concluded that the start-up case with constant acceleration was characterized by irregular behaviour and unstable while, in the impulsive case, a regular development of the spiral vortex could be recognized even in the self-similar stage. Concerns may thus arise for the fact that it is common practice to introduce regularization procedures in discrete-vortex numerical simulations in order to damp instabilities that most authors address to numerical causes only, but which may sometimes reside in the initial conditions of the problem.

To better assess the initial stages of this physical problem, we present here some accurate numerical simulations of the self-similar birth of a start-up vortex issuing from a semi-infinite flat plate computed by solving the Navier-Stokes equations in the streamfunction-vorticity formulation.

The main difficulty in the definition of a proper numerical algorithm for the integration of these equations is given by the need to limit the theoretically unbounded numerical domain and to provide a fast integration scheme in order to obtain sufficiently accurate unsteady solutions with reasonable computational time.

As a first step toward this goal the governing equations have been re-written with a proper nondimensionalization involving a "viscous" time scale. In the new variables the enlargement of the spiral with time is slower, thus allowing the introduction of a lower number of unknowns. Furthermore the accuracy requirement to avoid numerical smearing of the computed solution led to the development of a new and particularly efficient integration algorithm. The scheme is implicit, 2nd order accurate in both time and space coordinates. The number of unknowns is minimized by managing an unstructured grid of square cells. Grid points are introduced only in the region of non-negligible vorticity and the streamfunction on the consequent time-dependent numerical boundary is computed by using the Green integral. The implicit formulation implies a large linear system to be solved at each time step; this task is performed by a multigrid technique properly adapted to the unstructured grid. A special restriction algorithm increases the smoothing factor to a value of 9 , thus allowing convergence to be obtained at each time step in 4-5 multigrid cycles only. Through this algorithm, simulations with different scales of length and mesh sizes have been made possible and are going to be shown after the presentation of the mathematical formulation of the problem and the numerical algorithm. Finally, the viscous solutions have been compared with the results obtained by using an inviscid lumped-vortex method, thus revealing an interesting common behaviour that will be discussed in detail.

\section{The physical problem and the numerical algorithm}

The evolution of the viscous start-up spiral vortex can be described by four main steps:

1. Rayleigh stage: the flow is potential everywhere except for a thin Rayleigh-type viscous layer with constant thickness around the body;

2. Viscous stage: the convective terms of Navier-Stokes equations become comparable with viscous terms near the trailing edge where a vortex structure starts to appear;

3. Self-similar inviscid stage: convective terms become dominant, but the vortex is still small enough to be independent of geometry except for the trailing edge wedge angle;

4. Vortex expulsion: the reattachment and detachment points collide, the initial recirculation bubble opens up and the vortex starts lagging behind the airfoil.

ESAIM: Proc., Vol. 7, 1999, 248 -257 
In the present paper we undertake the simulation of the first three stages by solving the 2D incompressible Navier-Stokes (NS) equations in a cartesian domain $(x, y)$ around a semi-infinite flat plate $(y=0, x<0)$ in a streamfunction $\psi(x, y, t)$, vorticity $\omega(x, y, t)$ formulation:

$$
\nabla^{2} \psi=\omega ; \quad \omega_{t}+\psi_{y} \omega_{x}-\psi_{x} \omega_{y}=\nu \nabla^{2} \omega
$$

A no slip condition is imposed on the plate while in the far field the streamfunction approaches the limiting inviscid solution around an infinite wedge:

$$
\psi_{y}\left(x, 0^{ \pm}, t\right)=\psi\left(x, 0^{ \pm}, t\right)=0 \quad \forall x<0 ; \quad \lim _{r \rightarrow \infty} \psi=\psi_{\infty}=A t^{a} r^{m} \cos (m \theta) .
$$

The coefficient $m$ is related to the wedge angle $\beta=\pi(2-1 / m)$ and is equal to 0.5 in the case of a flat plate, $(r, \theta)$ are polar coordinates. With the assumed time variation of the potential far field velocity, a self-similar behaviour of the inviscid solution is allowed.

Since the initial stage of the viscous vortex roll-up is characterized by the formation of a Rayleigh type flow around the plate it is straightforward to use the Rayleigh layer thickness as a reference length for non-dimensionalization:

$$
R=(k \nu t)^{1 / 2} ; \quad x=\bar{x} R ; \quad y=\bar{y} R ; \quad \frac{1}{k}=a+\frac{m}{2} ; \quad \psi=\nu \bar{\psi} ; \quad \omega=\frac{\nu}{R^{2}} \bar{\omega} ; \quad t=t_{\nu} \bar{t}^{k} ;
$$

with $t_{\nu}$ a reference time related to the viscous time scale. Hence the non-dimensional form of the Navier-Stokes equations can be easily derived by inserting relations (3) into equations (1-2):

$$
\begin{aligned}
& \bar{\nabla}^{2} \bar{\psi}=\bar{\omega} ; \quad \quad \bar{t} \bar{\omega}_{\bar{t}}+\left[\left(\bar{\psi}_{\bar{y}}-\frac{k}{2} \bar{x}\right) \bar{\omega}\right]_{\bar{x}}+\left[\left(-\bar{\psi}_{\bar{x}}-\frac{k}{2} \bar{y}\right) \bar{\omega}\right]_{\bar{y}}=\bar{\nabla}^{2} \bar{\omega} ; \\
& \bar{\psi}_{\bar{y}}\left(\bar{x}, 0^{ \pm}, \bar{t}\right)=\bar{\psi}\left(\bar{x}, 0^{ \pm}, \bar{t}\right)=0 \quad \forall \bar{x}<0 ; \quad \lim _{\bar{r} \rightarrow \infty} \bar{\psi}=\bar{\psi}_{\infty}=\bar{t} \bar{r}^{m} \cos (m \bar{\theta}) .
\end{aligned}
$$

After this viscous transformation the only parameters left are $m$, related to the geometry and $a$ (appearing through the constant $k$ ), related to the type of unsteady start-up. In fact $a=0$ specifies a step-velocity, $a=1$ a constant acceleration and $a=\infty$ an infinitely smooth $\left(C_{\infty}\right)$ exponential start-up.

The viscous time scale is here derived by having the Reynolds number equal 1 at $r=R$. The value of the physical constant $A$ can be determined from the actual geometry by computing the leading term of the complex inviscid potential at the trailing edge in terms of the free-stream velocity $\left(V_{\infty}\right)$, the angle of attack $(\alpha)$ and the chord length $(c)$ of an actual airfoil.

Owing to the reference length's growth with time, in the new coordinate system the growth of the vortex is reduced and, especially for large values of the time, the number of the numerical unknowns is strongly limited, making the present mathematical formulation well-adapted to a numerical solution.

The computational mesh is unstructured and built with square cells of constant size $h$. Grid points are dynamically introduced during the computation in regions of non-negligible vorticity and are deleted if it becomes negligibly small. The Poisson equation is discretized by the usual 2nd order central difference operator. In the vorticity-transport equation, the velocity components are computed from the free-stream function explicitly determined by Adams time advancement. The discretization of the convective part is implicit and different than the usual explicit Adams 
time advancement. In addition an upwind bias is introduced in the implicit part of vorticity equation and compensated for in the explicit part, so as to obtain a good conditioning of the implicit linear system without destroying 2 nd order accuracy.

Finally, the time derivative and the diffusion term are discretised by a classical Crank-Nicolson scheme.

Whereas the streamfunction is known on the plate $(\psi=0)$, it is necessary to determine its value on the rest of the boundary in order to close the problem for the Poisson equation. It can be computed by using the Green integral:

$$
\begin{gathered}
\bar{\psi}(\bar{x}, \bar{y}, \bar{t})=\bar{t} \Re\left(\bar{z}^{m}\right)-\frac{1}{2 \pi} \int_{S} \bar{\omega}\left(\bar{x}_{0}, \bar{y}_{0}, \bar{t}\right) G\left(\bar{z}, \bar{z}_{0}\right) d S_{0} \\
G\left(\bar{z}, \bar{z}_{0}\right)=-\ln \left(\left|\frac{\bar{z}^{m}-\bar{z}_{0}^{m}}{\bar{z}^{m}-\left({\overline{z_{0}}}^{m}\right)^{*}}\right|\right), \quad \bar{z}=\bar{x}+i \bar{y} .
\end{gathered}
$$

Finally on the plate the no-slip condition is enforced by using Thom's relation for the vorticity [11].

The linear system obtained by writing the difference expressions of streamfunction-vorticity equations for each inner cell is simultaneously solved for the unknowns $(\bar{\psi}, \bar{\omega})$ by a V-cycle, linear multigrid technique with one pre/post-smoothing.

The smoothing algorithm is a red-black point relaxation. This is very simple and its smoothing properties are equivalent to any other's for the present problem. In a classical full multigrid V-cycle the smoothing factor of red-black is of the order of $1 / 3$ meaning that the error norm is reduced by a factor of approximately $1 / 3$ for each complete cycle, but this factor can be increased to $1 / 9$ with the modification proposed in [12] to which we refer for a description of the algorithm with application to the solution of the Poisson equation in a structured cartesian mesh. The convergence speed improvement is essentially due to a modification of the restriction stage with the available residual on the finer grid which is equivalent to an additional red-black smoothing. In order to apply multigrid to the present problem the main development effort was required for the management of the unstructured mesh, because of the unpredictable shape of the grid.

After the computation of all variables at time $\bar{t}^{(n+1)}$, an analysis of the flow at the external boundary allows us to re-build the grid for the next time step. The local value of vorticity is compared with a given threshold: if it is higher, the former boundary cell is completely encircled with new cells with zero vorticity and stream function obtained from the Green integral, if the value is lower and this is also true for all existing neighbouring cells the grid point is deleted.

\section{Results}

Calculations were performed using different mesh sizes; they exhibited a substantial agreement in behaviour. In Figure 1 the results obtained on the finest tested mesh $(h=0.1)$ are presented. The solution at four different times is shown in the case of impulsive acceleration of the plate to a constant speed $(a=0)$. The only numerical parameter of the computational domain is given by the non-dimensional length of the plate that is $L_{n}=38.4$ (the Rayleigh layer thickness being taken as reference length). The color logarithmic scale used for visualising the vorticity distribution displays the evolution of the start-up vortex. As anticipated, the initial stage of the motion is characterized by the presence of a Rayleigh flow on the whole plate with a constant boundary layer thickness; in Figure 1(a), at $\bar{t}=35.37$ the vorticity layer thickness is still constant on most of 
the plate (in all pictures only part of the actual plate is displayed) and the vortex is just starting to form. At $\bar{t}=561.1$, Figure 1(b), a regular spiral is clearly visible while on the lower side of the plate a region of negative vorticity evolves, revealing the reverse boundary layer induced by the primary separation at the plate apex. Perturbations start to appear when two turns of the spiral are completely developed, $\bar{t}=1298$, Figure $1(\mathrm{c})$. Subsequently chaotic motion dominates the flow even if a main spiral vortex is still recognizable, Figure $1(\mathrm{~d}), \bar{t}=1584$. The finite regions of higher vorticity clearly resemble the structures characterising unstable vortex sheets.

Substantially similar results are obtained from the simulation of the constant acceleration case $(a=1)$ presented in Figure 2 (same $h$ and $\left.L_{n}\right)$. The analysis confirms the development of a chaotic spiral vortex with a slight difference in core size and evolution of the spiral turns.

In both tests the number of cells is as large as 100,000 during the final part of the simulation. The CPU time bottle-neck of these simulations is given by the time required for the calculation of the Green integral in the external boundary cells, which has a non-linear growth versus the number of grid points $\left(N^{2}\right)$ while the internal integration is still very fast. There is, therefore, room for improvement. A main concern in this kind of numerical simulations is to understand if the vorticity spots developing in the solution are really a feature of the physical problem or, on the contrary, are driven by a lack of resolution in the numerical scheme or in the adopted mesh [13]. We repeated the simulations by using different mesh sizes; the structure of the computed sub-vortices appeared to be independent of the mesh size (at least for the finer grid computations) making us confident on the accuracy of the numerical solutions.

The present results can be usefully compared with Pierce's experimental results on the evolution of start-up spiral vortices around wedges of different shapes. Although a quantitative comparison is not possible due to the different shapes of the geometry, the shadowgraph images show streaklines of the flow which look impressively similar to the visualization of our numerical simulations. In both cases the presence of intermittent spots of vorticity is accompanied by a growing complexity of the flow with time.

Pullin and Perry studied the same phenomenon by a different apparatus, in order to compare the experiments with the numerical inviscid self-similar solution of Pullin. They could not completely verify the stability of the spiral vortex start-up since most of their results do not show regular and stable spirals. Anyway, having obtained a few pictures with a smooth spiral and a shape comparable to their numerical results, they conjectured the stability of the self-similar solution proposing apparatus vibrations as a possible source of the irregular experimental behaviour.

A different interpretation can be derived with the help of the present numerical simulation. The main difference between Pierce's and Pullin and Perry's experiments is given by the test Reynolds number. Due to the lack of a natural reference length in the problem, the Reynolds number as defined by Pullin and Perry is a function of time, strictly connected with our dimensionless time variable :

$$
\operatorname{Re}_{S}=\frac{A^{2 /(2-m)} t^{2(1+a) /(2-m)-1}}{\nu}
$$

Whereas in the experiments of Pullin and Perry $R e_{S}$ was of the order of $10^{3}$, in the tests of Pierce $R e_{S}$ ranged in the interval $\left(10^{4}-10^{5}\right)$; this difference, noted but not highlighted in the original papers, has a large impact on the results. We numerically reproduced the experiment of Pullin and Perry that better approximated a flat plate flow $(a=0.45, \beta=5 \mathrm{deg})$ and verified that the time scale of the experiment was equivalent to the viscous stage of our numerical simulation. The numerical results are even smoother than the experimental ones. This could be expected since the double-precision numerical simulation has clearly a much lower background noise than any 


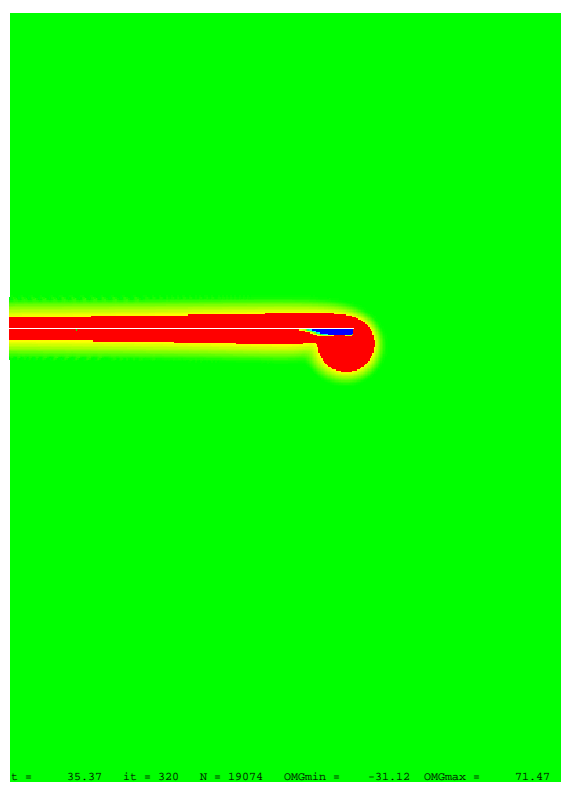

(a)

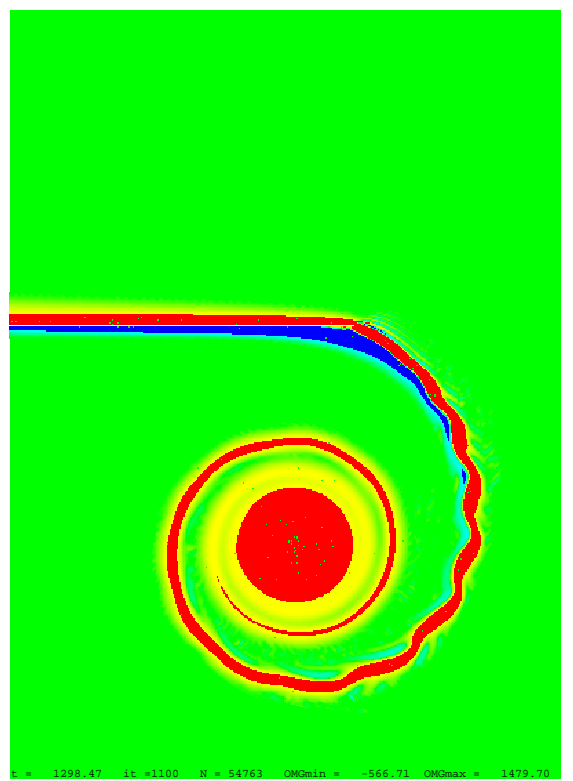

(c)

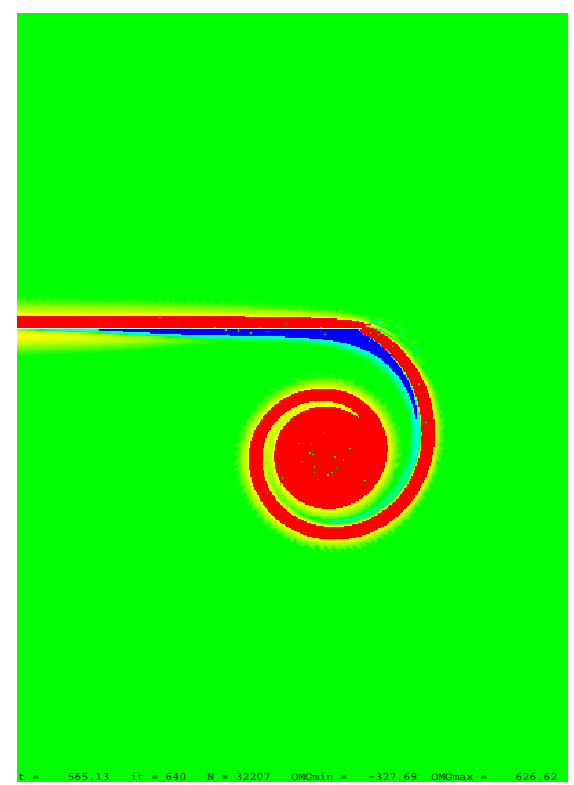

(b)

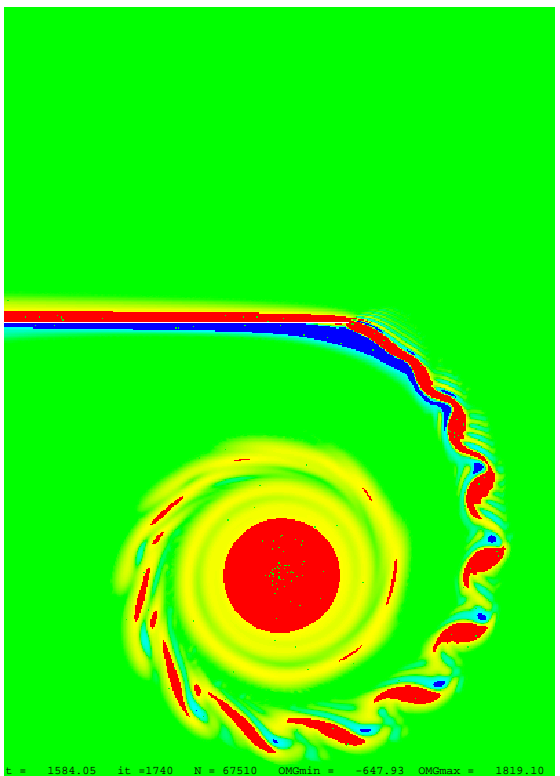

(d)

Figure 1: Solution at 4 different times of the impulsive start-up case $(a=0)$. Vorticity field represented by a color logarithmic scale.

(a): $\bar{t}=35.57$; time iteration $=320 ;$ number of grid cells $=19074 ; \bar{\omega}_{\min }=-31.12 ; \bar{\omega}_{\max }=71.47$.

(b): $\bar{t}=565.1$; time iteration $=640$; number of grid cells $=32207 ; \bar{\omega}_{\min }=-327.7 ; \bar{\omega}_{\max }=626.6$.

(c): $\bar{t}=1298$; time iteration $=1100$; number of grid cells $=54763 ; \bar{\omega}_{\min }=-566.7 ; \bar{\omega}_{\max }=1480$.

$(\mathrm{d}): \bar{t}=1584$; time iteration $=1740 ;$ number of grid cells $=67510 ; \bar{\omega}_{\min }=-647.9 ; \bar{\omega}_{\max }=1819$. 


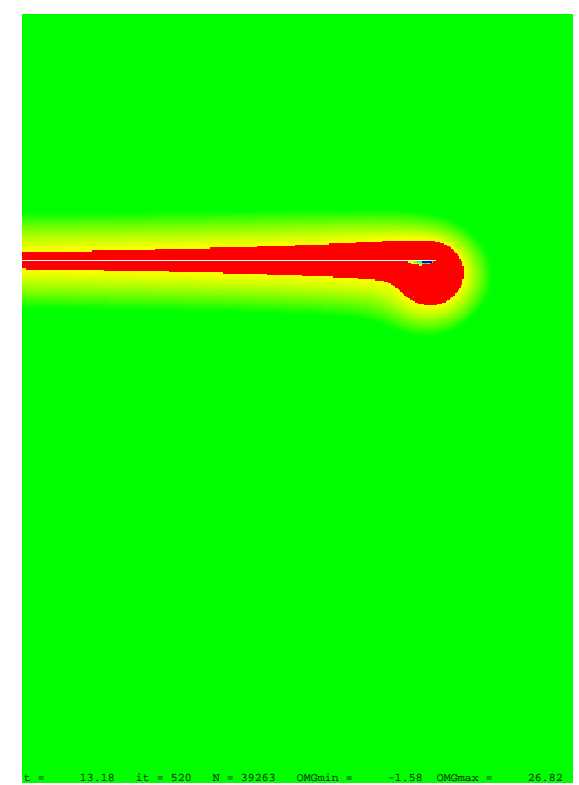

(a)

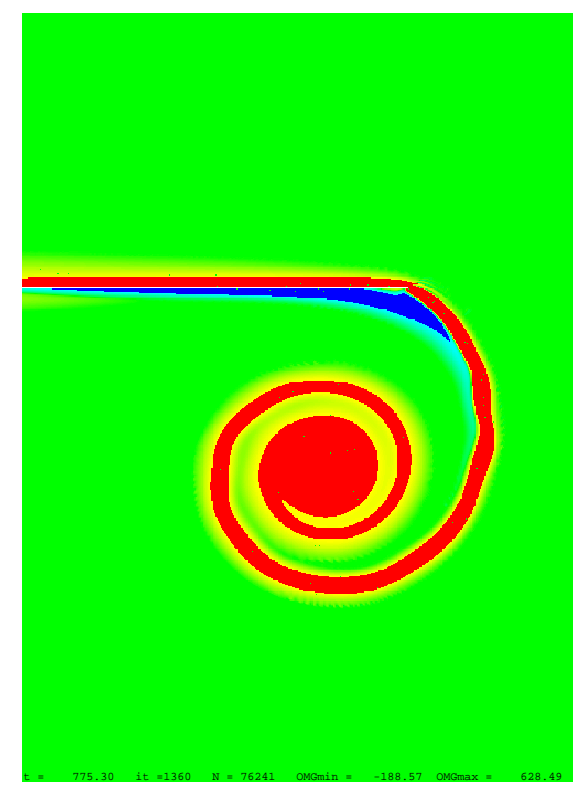

(c)

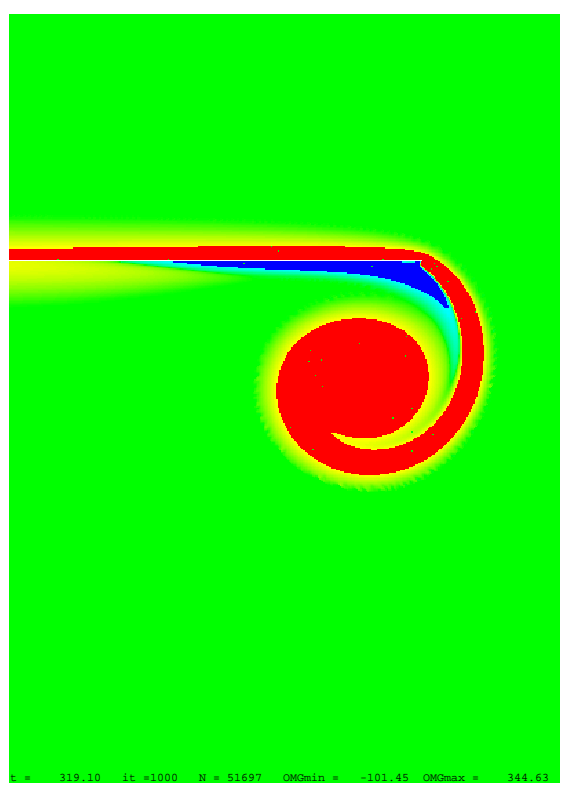

(b)

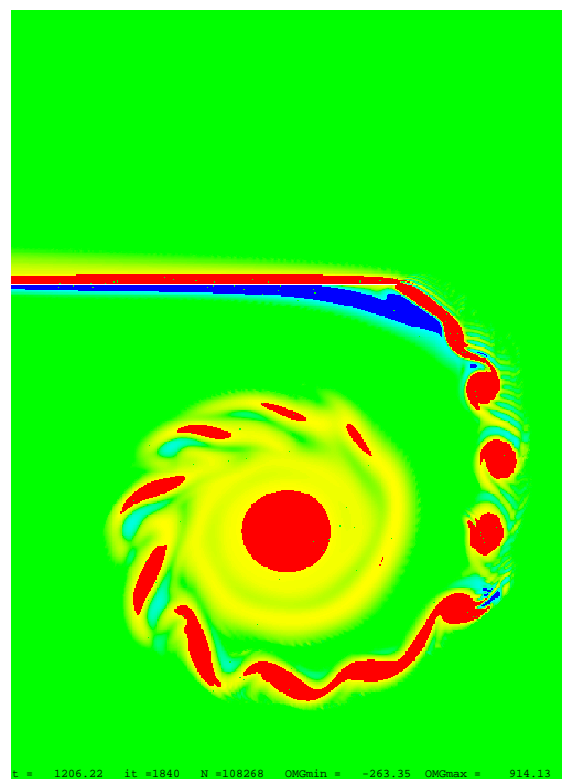

(d)

Figure 2: Solution at 4 different times of the impulsive start-up case $(a=1)$. Vorticity field represented by a color logarithmic scale.

(a): $\bar{t}=13.18$; time iteration $=520$; number of grid cells $=39263 ; \bar{\omega}_{\min }=-1.58 ; \bar{\omega}_{\max }=26.82$.

(b): $\bar{t}=319.1$; time iteration $=1000$; number of grid cells $=51697 ; \bar{\omega}_{\min }=-101.4 ; \bar{\omega}_{\max }=344.6$.

(c): $\bar{t}=775.3$; time iteration $=1360$; number of grid cells $=76241 ; \bar{\omega}_{\text {min }}=-188.6 ; \bar{\omega}_{\text {max }}=628.5$.

(d): $\bar{t}=1206$; time iteration $=1840$; number of grid cells $=108268 ; \bar{\omega}_{\min }=-263.3 ; \bar{\omega}_{\max }=914.1$. 
practical experiment.

A concluding remark on the comparison with the experiments is that our NS simulations do not reveal any formation of a secondary spiral vortex with opposed sign circulation. This is in agreement with Pullin and Perry's results that show the birth of a secondary vortex only for the larger values of the wedge angle $\beta$.

In the already cited work of Koumoutsakos and Shiels [10] the viscous simulation of the start-up of a plate of finite thicknes was performed by a "blob" method. While their results are in agreement with the present ones for the case $a=1$, for the impulsive case $a=0$ Koumoutsakos and Shiels propose a regular vortex spiral evolution. However a comparison of the results needs some care. The simulation of a plate of semi-infinite thickness gave us the possibility to concentrate on the self-similar stage of the spiral vortex evolution while Koumoutsakos and Shiels presented a much more complex phenomenon including the vortex expulsion phase, with an unavoidable lack of resolution in the self-similar stage. In fact we extended the self similar vortex computation up to $\bar{t}=1000-2000$, whereas in the simulation [10] the self-similar phase ended at $\bar{t} \simeq 200$. Hence it is possible that in these simulations the instabilities had not the time to evidence because the vortex already started lagging behind the plate.

The results of our NS simulations can be compared against the unsteady inviscid simulations obtained by solving the Euler equations around the same domain in a coordinate system composed of Prandtl's similarity variables plus time [7]. The inviscid solution is modelled by a lumped vortex discretization of the flow field.

The discrete equations are integrated in time by a 2nd order Runge-Kutta algorithm with the streamfunction analytically computed by conformal mapping and an analytically imposed Kutta condition of finite velocity at the wedge's apex. In order to limit the number of vortices adopted in the simulation, after each time step the last vortex defining the spiral accumulates in a barycentre. The computation was run until the obtained solution reached a statistically steady state (in the sense that average quantities such as the total vorticity ceased to drift). Two snapshots of the solutions thus obtained are proposed in Figure 3 for the case $m=0.5$ (flat plate) with $a=0$ and $a=1$. In the picture the radius of the displayed circles are proportional to the intensity of the point vortices. Their distribution is randomly oscillating about a recognizable spiral structure, more clearly defined for $a=0$. Despite these random motions, an "average" self-similarity exists because the size of the spiral in the $\mathrm{z}$ plane and the total circulation measured in similarity variables do not exhibit any noticeable oscillations with time. The numerical discretization did not affect the results, the solutions are not sensitive to the time step and to the number of discrete vortices if an adequate number of vortices and a sufficiently small time step are used.

In order to compare these inviscid unsteady solutions with the results of the NS simulations, we present in Figure 4, for $a=0$ (similar results for $a=1$ have been obtained), the average shape of the spiral vortices obtained by plotting the time averaged vorticity in the inviscid self-similar coordinate planes. The agreement is good: the viscous and inviscid average spiral vortices are practically coincident in both size and shape.

While addressing the interested reader to [14] for a more extended and quantitative analysis of the results, another question posed in the introduction can now be answered. The present NS numerical simulations, in substantial agreement with the experiments, suggest that the birth of a start-up vortex is characterized by unstable motion once it enters the inviscid self-similar-on-themean stage. This evolution is also well described by unsteady inviscid lumped-vortex methods that correctly mimic the chaotic behaviour of the flow. Therefore, there does not seem to be any reason to prefer smooth inviscid solutions for the purpose of describing vortex start-up. Many efforts have been devoted to the development of regularization procedures in discrete vortex methods to preserve smoothness of the results [15]. Krasny $[16,17]$ proved by a high accuracy-numerical 


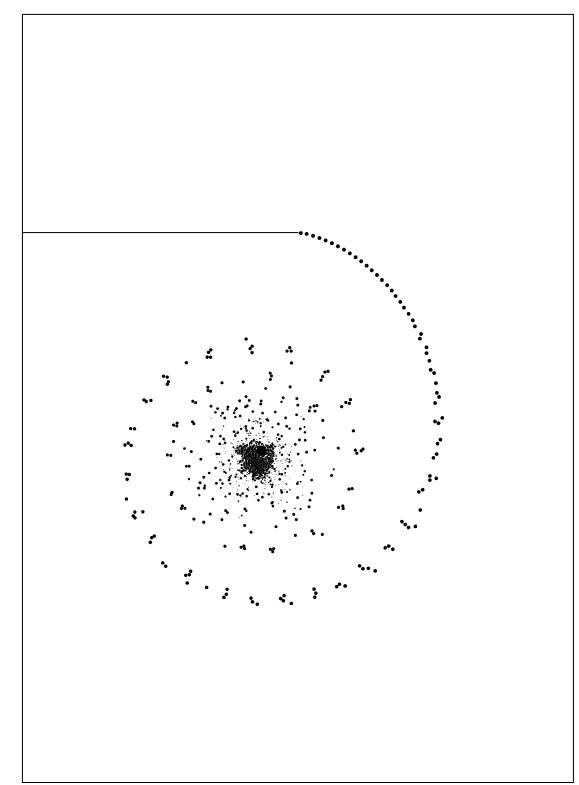

(a)

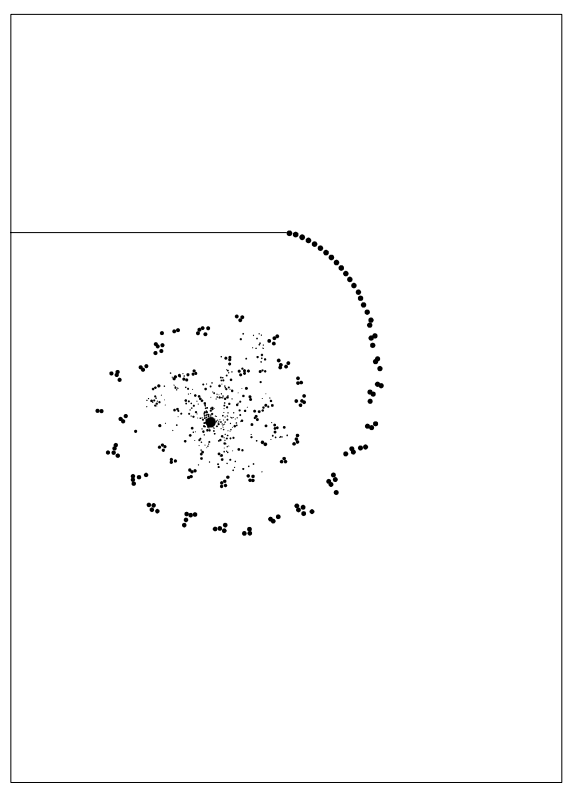

(b)

Figure 3: Vortex spirals computed by the unsteady lumped-vortex method. The radius of the circles is proportional to the value of the local circulation. (a): case $a=0 ; N=1300$; number of time steps $=7500 ;$ (b): case $a=1 ; N=500$; number of time steps $=10000$.

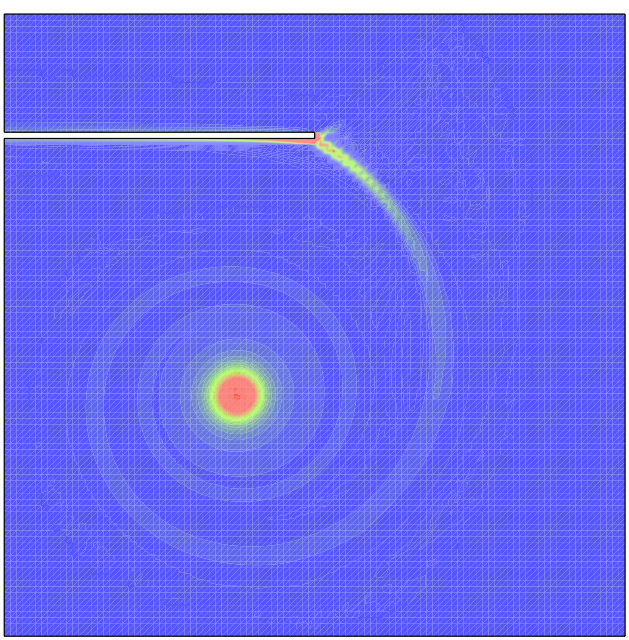

(a)

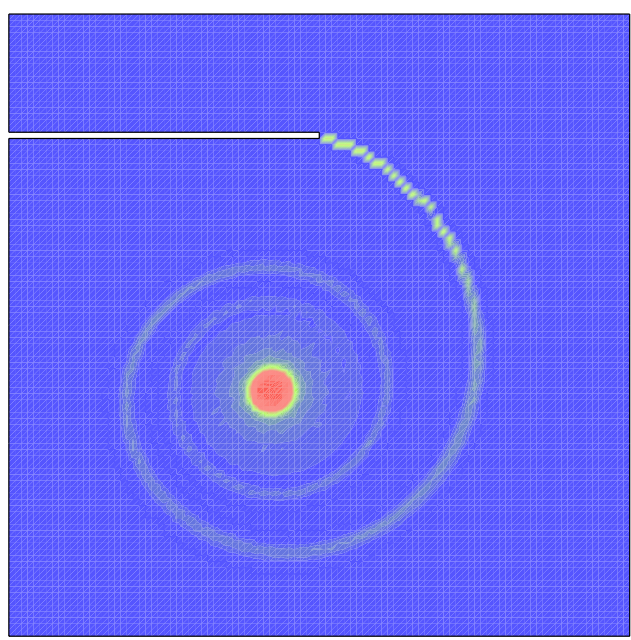

(b)

Figure 4: Average vorticity $\bar{\Omega}(\xi, \eta)$ computed in inviscid self-similar variables; case $(a=0)$. Representation by a color scale with range $(0,500)$. (a): Navier-Stokes solution. (b): Inviscid unsteady solution. 
method that the inviscid evolution of an initially continuous vortex sheet requires a non zero time to develop a singularity. However, this result has no bearing on the present problem because at time zero vorticity is not concentrated in a vortex sheet but in a single point. Therefore there is no guarantee that the inviscid solution will be regular for any time interval. On the contrary, the agreement between the unstable behaviour of the NS simulation and that of the unsteady Euler simulation shows that a regularization in an inviscid discrete vortex method could tend to overstabilize a problem that is unstable in nature. Whereas such a procedure is necessary, for instance, to determine the initially smooth evolution of an infinite vortex sheet, there is an important difference between an infinite vortex sheet and the start-up vortex: the initial singularity at the wedge from which the latter evolves.

\section{Conclusions}

A numerical method has been presented for the two-dimensional simulation of the start-up vortex issuing from a semi-infinite flat plate accelerated from rest in still air. The algorithm solves the $\psi-\omega$ formulation of the incompressible Navier-Stokes equations. Particular care has been devoted to the development of an efficient numerical method. The number of grid cells is minimized by using a non-dimensionalization of the flow equations based on the Rayleigh layer thickness and a viscous time scale; moreover an unstructured grid with square cells is dynamically defined at each time step only in regions of non-negligible vorticity. An implicit discretization has been proposed with a fast simultaneous integration of the streamfunction and vorticity equations by a multigrid technique with a smoothing factor of $1 / 9$.

Two simulations have been presented: uniform and impulsive acceleration of the plate. In both cases the viscous solution starts to exhibit oscillations as soon as a recognizable vortex spiral begins to appear, although the vortex evolution follows the inviscid similarity rule in terms of global parameters (core position, vortex size, total circulation). The evolution of the oscillations is similar to the Kelvin-Helmholtz instability of a plane vortex sheet. These results provide a deeper insight on a phenomenon which is still debated and not completely clarified by experimental evidence. The qualitative agreement of the numerical simulations with the results of Pierce's experiments is noteworthy. On the other side, our calculations suggest that Pullin and Perry's experimental results are not in disagreement with Pierce's: the discrepancies are due to a difference in Reynolds number (or equivalently in time scale). In Pullin and Perry's tests the vortex spirals are in an earlier stage in which viscous effects are comparable with the convective ones whereas Pierce's results show a later stage in which a mainly inviscid flow has become unstable.

This conclusion also sheds a different light on the capability of inviscid lumped-vortex methods to properly describe inviscid phenomena. In fact, the average vorticity distribution produced by the NS calculation, when plotted in the similarity plane, is in a remarkable agreement, as far as the total circulation and core position, with the results obtained by an unsteady inviscid simulation with discrete point vortices. In addition, the inviscid numerical solution is characterized by an irregular evolution with agglomerations of point vortices which behave just like the vorticity spots present in the NS simulations. Smooth inviscid spirals, similar to those obtained by previous authors who looked for time-independent solutions, do not seem to be preferable to the chaotic, although coherent on the average, solutions of the point-vortex equations. Hence regularization in discrete vortex simulations of trailing vortices is not always recommended and may be even dangerous. In fact the irregular motion is a property of the physical system since the very first instant in which an inviscid description makes sense, and not only of its point-vortex numerical approximation. 


\section{References}

1 Saffman, P.G.: Vortex Dynamics. Cambridge University Press, Cambridge (1992).

2 Rotт, N.: Diffraction of a Weak Shock with Vortex Generation. J. Fluid. Mech., 28 (1956), 111-128.

3 Moore, D.W.: The Stability of an Evolving Two-Dimensional Vortex Sheet. Mathematika, 23 (1976), 35-44.

4 Pullin, D.I.: The Large-Scale Structure of Unsteady Self-Similar Rolled-Up Vortex Sheets. J. Fluid. Mech. 88 (1978), 401-430.

5 Pierce, D.: Photographic Evidence of the Formation and Growth of Vorticity behind Plates Accelerated from Rest in Still Air. J. Fluid. Mech. 11 (1961), 460-464.

6 Pullin, D.I. And Perry, A.E.: Some Flow Visualization Experiments on the starting Vortex. J. Fluid. Mech. 97, part 2 (1980), 239-255.

7 Luchini, P.: The Self-Similar Birth of a Startup Vortex. Oral communication at the I European Fluid Mechanics Conference, Cambridge (1991).

8 Luchini, P.: Evolution of a Startup vortex from an Airfoil. Atti dell'XI Congresso Nazionale AIMETA, Meccanica dei Fluidi, Trento (Italy). (1992), 103-108.

9 Luchini, P.: The The Viscous Stage of Startup Vortex Shedding. Oral communication at the EUROMECH 305 and ERCOFTAC Workshop "Dynamics and Geometry of Vortical Structures", Cortona (Italy), (1993).

10 Koumoutsakos, P. And Shiels, D.: Simulations of the viscous flow normal to an impulsively started and uniformly accelerated flat plate. J. Fluid Mech., 328, (1996), 177-227.

11 Roache, P.J.: Computational Fluid Dynamics. Hermosa Publishers, Albuquerque (1972).

12 Luchini, P. And D'Alascio, A.: Multigrid Pressure Correction Techniques for the Computation of Quasi-Incompressible Internal Flows. J. Num. Meth. in Fluids, 18, (1994), 489-507.

13 Brown, D.L. And Minion, M.L.: Performance of Under-resolved Two-Dimensional Incompressible Flow Simulations. J. Comp. Physics, 122, (1995), 165-183.

14 Luchini, P. And Tognaccini, R.: The Strat-Up Vortex Issuing from a Semi-Infinite Flat Plate. Submitted for publication on Theor. Comp. Fluid Dyn.

15 Sarpkaya, T.: Computational Methods with Vortices - The 1988 Freeman Scholar Lecture. J. Fluids Eng. 111, (1989), 5-52.

16 Krasny, R.: Vortex sheet computations: Roll-up, Wakes, Separation. Lect. Applied Math, 28, (1991), 385-402.

17 Krasny, R.: A Study of Singularity Formation in a Vortex Sheet by the Point-Vortex Approximation. J. Fluid. Mech., 167, 65-93. 